\title{
Étude du formage superplastique de couvercles en 3D par la méthode des sections critiques
}

\author{
Eugène Chumachenko ${ }^{1}$, Thomas Billaudeau² et Alexandre Piard ${ }^{3 a}$ \\ 1 Moscow State Institute of Electronics and Mathematics (Technical University), Dep. of Mathematical Simulation, \\ 3/12, B. Trehsviatitelskiy per., 109028 Moscow, Russia \\ 2 AIRBUS France, Materials and Processes, 316 route de Bayonne, 31931 Toulouse Cedex 9, France \\ 3 SOGETI HIGH TECH, Consulting, 1 chemin de la Crabe, 31025 Toulouse Cedex, France
}

Reçu le 7 mars 2005 ; accepté le 31 mai 2006

\begin{abstract}
Résumé - Ce travail étudie le problème de la simulation mathématique du formage superplastique. Un algorithme original a été développé afin de permettre la résolution du système provenant de la méthode des éléments-finis, en se basant sur les sections orthogonales critiques. Des études expérimentales ont été menées et ont permis l'estimation de la corrélation entre les prévisions numériques et les données expérimentales. Les résultats obtenus et les algorithmes retenus permettent d'améliorer de façon substantielle l'efficacité et la rapidité du travail pour la conception de nouveaux procédés et la modernisation des procédés de formage dans des conditions de superplasticité. Le matériau utilisé est un TA6V.
\end{abstract}

Mots clés : Simulations numériques / superplasticité / formage / optimisation / section orthogonale critique

\begin{abstract}
A 3D study of the superplastic forming of lids by the method of the critical sections. While developing the technological processes for isothermal gas forming of the complicated shape shells, it is very important to have the information about the progression of the shape changes, and to be able to predict thinning of a shell and filling of die cavities. These problems are solved using computer simulation. Creating a complex 3-dimensional mathematical model for this simulation is not always justified as it leads to a significant labor cost resulting from both data preparation and subsequent calculations. In order to obtain the essential estimates for the above-mentioned technological processes and for the optimization of pressure and temperature parameters, it is more practical to use simplified 2.5D models. This allows analyzing significantly larger number of feasible parameter sets in search for the optimal solution. It turns out that in many cases these estimates are practically acceptable except for extremely complex die patterns. For the most critical parts, after getting an initial estimate solution, one can carry out additional calculations utilizing a full 3D model. This approach reduces to a minimum the number of prototypes of forming trials. In this paper, we introduce a new technique estimating progressive shape changes for 3-dimensional shells based on the generalized cross-sections method.
\end{abstract}

Key words: Numerical simulations / superplastic forming / optimisation / critical orthogonal sections

\section{Introduction}

Le développement du formage superplastique, pour des pièces de formes complexes, nécessite la parfaite connaissance de l'évolution de la forme de la tôle, au cours $\mathrm{du}$ formage, ainsi qu'une bonne prédiction de l'amincissement de la tôle. Ces problèmes sont traités à l'aide de la simulation numérique du procédé.

\footnotetext{
a Auteur correspondant : alexandre.piard@sogeti.com
}

La création d'un modèle mathématique 3D complet pour la simulation n'est pas forcément le plus justifié car il conduit à des études laborieuses et coûteuses, autant pour la phase de caractérisation des paramètres que pour les calculs proprement dits. Dans le but d'obtenir les prédictions essentielles pour ces procédés et pour l'optimisation de la pression et de la température, il est plus pratique d'utiliser des modèles 2,5D simplifiés. Ces modèles permettent en effet d'analyser un grand nombre de paramètres lors de la phase de recherche de 


\section{Nomenclature}

\begin{tabular}{|ll|}
\hline$\sigma_{x y}$ & Tenseur de contrainte. \\
$\varepsilon_{x y}$ & Tenseur de déformation. \\
$\dot{\varepsilon}_{x y}$ & Tenseur des vitesses de déformation. \\
$u_{1}, u_{2}, u_{3}$ & Vitesses de déplacement. \\
$c_{0} A$ et $a$ & Constantes caractérisant l'inclinaison dans le plan $(z, x)$. \\
$c_{0} B$ et $b$ & Constantes caractérisant l'inclinaison dans le plan $(z, y)$. \\
$c_{0} C$ et $c$ & Constantes caractérisant le déplacement le long de $Z$. \\
$\dot{\theta}$ & Vitesse de changement de volume. \\
$K$ & Facteur de compression triaxiale. \\
$\mu$ & Facteur apparent de viscosité, fonction de l'intensité de la vitesse de déformation. \\
$\sigma^{*}$ & Pression hydrostatique accumulée. \\
$l_{0}$ & Dimension initiale caractéristique de la section. \\
$l$ & Longueur de la ligne moyenne du couvercle, à la section considérée, après formage. \\
$P(t)$ & Pression exercée. \\
$l_{i}^{0}$ & Longueur initiale de la section $i$ du couvercle. \\
$l_{i}$ & Longueur du pourtour correspondant à la section de la matrice. \\
$h$ & Hauteur de la pièce. \\
\hline
\end{tabular}

solution optimale. Il s'avère que ces estimations sont bonnes dans la plupart des cas sauf pour des outils de formes extrêmement complexes. Pour ces cas critiques, après avoir obtenu une estimation initiale, le recours à des calculs supplémentaires avec le modèle 3D permet d'avoir une idée plus précise des estimations. Cette approche réduit au minimum le nombre de prototypes d'essais de formage.

Cet article présente une nouvelle technique d'estimation du changement de forme de pièces tridimensionnelles basée sur la méthode des coupes transversales.

\section{Mise au point d'une solution par approximation}

Dans l'analyse du formage superplastique de pièces tridimensionnelles à l'aide de sections planes, on suppose que la dimension caractéristique dans le sens de la section orthogonale $(x, y)$ n'est pas inférieure à la dimension caractéristique de la section elle-même (Fig. 1).

Soit, pour chaque valeur définie $z=$ const., prise au coeur de la déformation le long de l'axe $Z$, nous avons simultanément deux groupes d'équivalences :

le premier - pour l'élément tenseur de contraintes :

$$
\begin{aligned}
& \sigma_{11}=\sigma_{11}(x, y), \quad \sigma_{22}=\sigma_{22}(x, y), \quad \sigma_{12}=\sigma_{12}(x, y), \\
& \sigma_{13}=\sigma_{23}=0, \quad \sigma_{33}=\sigma_{33}(x, y) \text {; }
\end{aligned}
$$

le deuxième - pour l'élément tenseur de vitesse de déformation :

$$
\begin{aligned}
& \dot{\varepsilon}_{11}=\dot{\varepsilon}_{11}(x, y), \quad \dot{\varepsilon}_{22}=\dot{\varepsilon}_{22}(x, y), \quad \dot{\varepsilon}_{12}=\dot{\varepsilon}_{12}(x, y) \\
& \dot{\varepsilon}_{13}=\dot{\varepsilon}_{23}=0, \quad \dot{\varepsilon}_{33}=\dot{\varepsilon}_{33}^{0}(x, y) \cdot g(z) .
\end{aligned}
$$
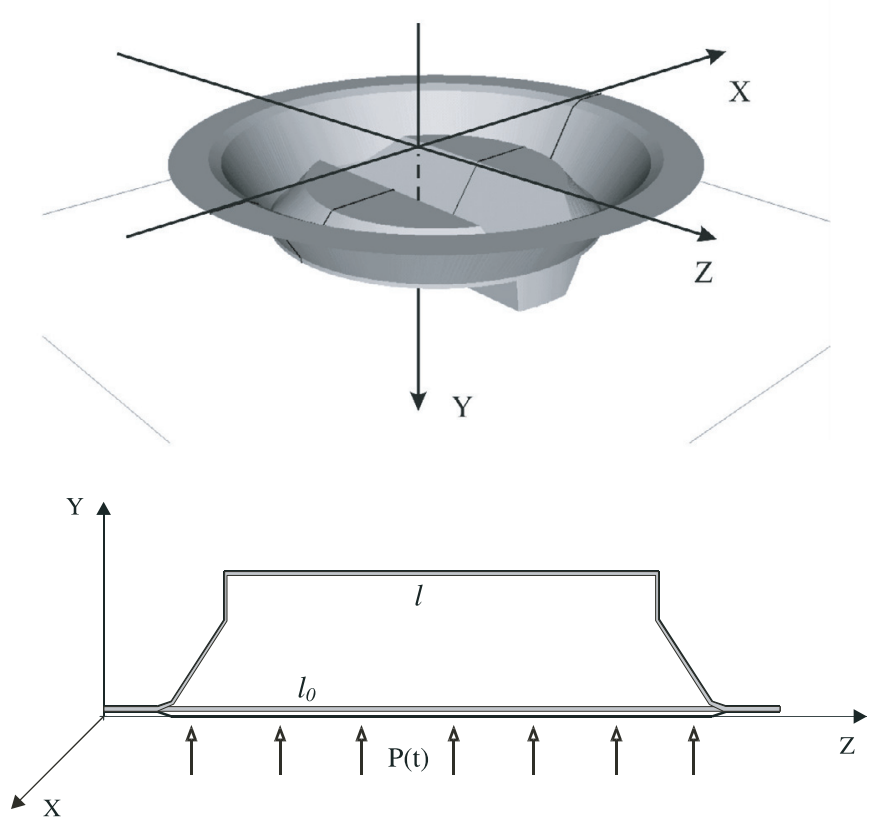

Fig. 1. Sélection du système de coordonnées.

Regardons les simplifications qui apparaissent dans les principales équations dans ce cas.

Soient $u_{1}, u_{2}, u_{3}$ - les vitesses de déplacement.

À partir de la condition :

$$
\dot{\varepsilon}_{33}=\frac{\partial u_{3}}{\partial z}
$$

il en découle que :

$$
u_{3}=\dot{\varepsilon}_{33}^{0}(x, y) \cdot\left(c_{0}+\int_{0}^{z} g\left(z^{\prime}\right) \mathrm{d} z^{\prime}\right)+f(x, y)
$$


et dans ce cas :

$$
\begin{aligned}
& \frac{\partial u_{3}}{\partial x}=\frac{\partial \dot{\varepsilon}_{33}^{0}(x, y)}{\partial x} \cdot\left(c_{0}+\int_{0}^{z} g\left(z^{\prime}\right) \mathrm{d} z^{\prime}\right)+\frac{\partial f(x, y)}{\partial x}, \\
& \frac{\partial u_{3}}{\partial y}=\frac{\partial \dot{\varepsilon}_{33}^{0}(x, y)}{\partial y} \cdot\left(c_{0}+\int_{0}^{z} g\left(z^{\prime}\right) \mathrm{d} z^{\prime}\right)+\frac{\partial f(x, y)}{\partial y}
\end{aligned}
$$

À partir de la condition :

$$
2 \dot{\varepsilon}_{13}=\frac{\partial u_{1}}{\partial z}+\frac{\partial u_{3}}{\partial x}
$$

il en découle que :

$$
\begin{aligned}
\frac{\partial u_{1}}{\partial z}= & -\frac{\partial u_{3}}{\partial x}= \\
& -\frac{\partial \dot{\varepsilon}_{33}^{0}(x, y)}{\partial x} \cdot\left(c_{0}+\int_{0}^{z} g\left(z^{\prime}\right) \mathrm{d} z^{\prime}\right)-\frac{\partial f(x, y)}{\partial x}, \quad(4) \\
u_{1}=-\frac{\partial \dot{\varepsilon}_{33}^{0}(x, y)}{\partial x} \cdot\left(c_{1}+c_{0} \cdot z+\int_{0}^{z} \mathrm{~d} z^{\prime \prime} \int_{0}^{z^{\prime}} g\left(z^{\prime}\right) \mathrm{d} z^{\prime}\right) & -\frac{\partial f(x, y)}{\partial x} z+q_{1}(x, y)
\end{aligned}
$$

De la même façon, à partir de la condition :

$$
2 \dot{\varepsilon}_{23}=\frac{\partial u_{2}}{\partial z}+\frac{\partial u_{3}}{\partial y}=0
$$

il en découle que :

$$
\begin{aligned}
\frac{\partial u_{2}}{\partial z}= & -\frac{\partial u_{3}}{\partial y}= \\
& -\frac{\partial \dot{\varepsilon}_{33}^{0}(x, y)}{\partial y} \cdot\left(c_{0}+\int_{0}^{z} g\left(z^{\prime}\right) \mathrm{d} z^{\prime}\right)-\frac{\partial f(x, y)}{\partial y}
\end{aligned}
$$

Ensuite, étant donné que :

$$
\begin{aligned}
& \frac{\partial u_{1}}{\partial x}=\dot{\varepsilon}_{11}(x, y), \quad \frac{\partial u_{2}}{\partial y}=\dot{\varepsilon}_{22}(x, y), \\
& \frac{\partial u_{1}}{\partial y}+\frac{\partial u_{2}}{\partial x}=2 \dot{\varepsilon}_{12}(x, y)
\end{aligned}
$$

nous obtenons à partir de la première équation (6) :

$$
\begin{aligned}
\dot{\varepsilon}_{11}(x, y)= & \frac{\partial u_{1}}{\partial x} \\
\dot{\varepsilon}_{11}(x, y)= & -\frac{\partial^{2} \dot{\varepsilon}_{33}^{0}(x, y)}{\partial x^{2}} \cdot\left(c_{1}+c_{0}+\int_{0}^{Z} \mathrm{~d} z^{\prime \prime} \int_{0}^{Z^{\prime \prime}} g\left(z^{\prime}\right) \mathrm{d} z^{\prime}\right) \\
& -\frac{\partial^{2} f(x, y)}{\partial x^{2}}+\frac{\partial q_{1}(x, y)}{\partial x}
\end{aligned}
$$

ce qui donne :

$$
\frac{\partial^{2} \dot{\varepsilon}_{33}^{0}(x, y)}{\partial x^{2}}=0 ; \quad \frac{\partial^{2} f(x, y)}{\partial x^{2}}=0
$$

À partir de la seconde équation (6) nous avons :

$$
\begin{aligned}
& \dot{\varepsilon}_{22}(x, y)=\frac{\partial u_{2}}{\partial y} \\
& \dot{\varepsilon}_{22}(x, y)=-\frac{\partial^{2} \dot{\varepsilon}_{33}^{0}(x, y)}{\partial y^{2}} \cdot\left(c_{2}+c_{0}+\int_{0}^{Z} \mathrm{~d} z^{\prime \prime} \int_{0}^{Z^{\prime \prime}} g\left(z^{\prime}\right) \mathrm{d} z^{\prime}\right) \\
& \quad-\frac{\partial^{2} f(x, y)}{\partial y^{2}}+\frac{\partial q_{2}(x, y)}{\partial y}
\end{aligned}
$$

et, étant donné que l'expression obtenue ne doit pas dépendre de $z$ :

$$
\frac{\partial^{2} \dot{\varepsilon}_{33}^{0}(x, y)}{\partial y^{2}}=0 ; \quad \frac{\partial^{2} f(x, y)}{\partial y^{2}}=0
$$

Et, enfin, à partir de la troisième équation (6), nous obtenons :

$$
\begin{aligned}
2 \dot{\varepsilon}_{12}(x, y)= & \frac{\partial u_{1}}{\partial y}+\frac{\partial u_{2}}{\partial x} \\
2 \dot{\varepsilon}_{12}(x, y)= & -\frac{\partial^{2} \dot{\varepsilon}_{33}^{0}(x, y)}{\partial x^{2}} \\
& \cdot\left(c_{1}+c_{2}+2 c_{0} z+2 \int_{0}^{Z} \mathrm{~d} z^{\prime \prime} \int_{0}^{Z^{\prime \prime}} g\left(z^{\prime}\right) \mathrm{d} z^{\prime}\right) \\
& -2 \frac{\partial^{2} f(x, y)}{\partial x \partial y} z+\frac{\partial q_{1}(x, y)}{\partial y}+\frac{\partial q_{2}(x, y)}{\partial x} \\
\frac{\partial^{2} \dot{\varepsilon}_{33}^{0}(x, y)}{\partial x \partial y}= & 0 ; \quad \frac{\partial^{2} f(x, y)}{\partial x \partial y}=0
\end{aligned}
$$

(6) À partir des équations (7)-(9) nous avons :

$$
f(x, y)=a x+b y+c, \quad \dot{\varepsilon}_{33}^{0}(x, y)=A x+B y+C
$$


Ainsi, la vue d'ensemble des déplacements peut, pour le cas examiné, être présentée de la manière suivante :

$u_{1}=-A \cdot\left(c_{1}+c_{0} \cdot z+\int_{0}^{z} \mathrm{~d} z^{\prime \prime} \int_{0}^{z^{\prime \prime}} g\left(z^{\prime}\right) \mathrm{d} z^{\prime}\right)-a z+q_{1}(x, y)$,

$u_{2}=-B \cdot\left(c_{2}+c_{0} \cdot z+\int_{0}^{z} \mathrm{~d} z^{\prime \prime} \int_{0}^{z^{\prime \prime}} g\left(z^{\prime}\right) \mathrm{d} z^{\prime}\right)-b z+q_{2}(x, y)$,

$u_{3}=(A x+B y+C) \cdot\left(c_{0}+\int_{0}^{z} g\left(z^{\prime}\right) \mathrm{d} z^{\prime}\right)+(a x+b y+c)$.

Notons que les constantes $c_{1}, c_{2}$ peuvent être rapportées aux fonctions aléatoires $q_{1}, q_{2}$ (écart d'un ensemble fixe sur les axes $\mathrm{O} x$ et $\mathrm{O} y$ ). Les constantes $c_{0} A$ et a caractérisent l'inclinaison, comme étant celle d'un ensemble fixe, dans le plan $(z, x)$. De la même façon, $c_{0} B$ et $b$ caractérisent l'inclinaison dans le plan $(z, y)$, et les constantes $c_{0} C$ et $c$, le déplacement le long de $Z$.

Si l'on néglige tous les déplacements qui sont caractéristiques du déplacement d'un corps pris comme ensemble fixe ou déplacement de corps rigide (en les ramenant à zéro), nous arrivons à la structure suivante pour les vitesses de déplacements :

$$
\begin{aligned}
& \left.u_{1}=-A \int_{0}^{z} \mathrm{~d} z^{\prime \prime} \int_{0}^{z^{\prime \prime}} g\left(z^{\prime}\right) \mathrm{d} z^{\prime}\right)+q_{1}(x, y), \\
& \left.u_{2}=-B \int_{0}^{z} \mathrm{~d} z^{\prime \prime} \int_{0}^{z^{\prime \prime}} g\left(z^{\prime}\right) \mathrm{d} z^{\prime}\right)+q_{2}(x, y), \\
& u_{3}=(A x+B y+C) \cdot \int_{0}^{z} g\left(z^{\prime}\right) \mathrm{d} z^{\prime} .
\end{aligned}
$$

Un contrôle direct permet de constater avec certitude que les expressions obtenues pour les vitesses de déplacements $u_{i}$ permettent la réalisation de (1), (2). Nous pouvons maintenant résoudre les équations (1) et (2) à l'aide des expressions des vitesses de déplacement $u_{i}$ ci-dessous.

En effet,

$$
\begin{aligned}
& \dot{\varepsilon}_{11}=q_{1, x}(x, y), \quad \dot{\varepsilon}_{22}=q_{2, x}(x, y), \\
& 2 \dot{\varepsilon}_{12}=q_{1, y}(x, y)+q_{2, x}(x, y), \\
& 2 \dot{\varepsilon}_{13}=(-A+A) \cdot \int_{0}^{z} g\left(z^{\prime}\right) \mathrm{d} z^{\prime}=0, \\
& 2 \dot{\varepsilon}_{23}=(-B+B) \cdot \int_{0}^{z} g\left(z^{\prime}\right) \mathrm{d} z^{\prime}=0, \\
& \dot{\varepsilon}_{33}=(A x+B y+C) \cdot g(z)
\end{aligned}
$$

Ensuite, la réalisation des équations de combinaison des déformations de Saint-Venant donne :

$$
\begin{aligned}
& \frac{\partial^{2} \dot{\varepsilon}_{11}}{\partial y^{2}}+\frac{\partial^{2} \dot{\varepsilon}_{22}}{\partial x^{2}}=2 \frac{\partial^{2} \dot{\varepsilon}_{12}}{\partial x \partial y} ; \\
& \frac{\partial^{2} \dot{\varepsilon}_{22}}{\partial z^{2}}+\frac{\partial^{2} \dot{\varepsilon}_{33}}{\partial y^{2}}=2 \frac{\partial^{2} \dot{\varepsilon}_{23}}{\partial y \partial z} ; \\
& \frac{\partial^{2} \dot{\varepsilon}_{33}}{\partial x^{2}}+\frac{\partial^{2} \dot{\varepsilon}_{11}}{\partial z^{2}}=2 \frac{\partial^{2} \dot{\varepsilon}_{31}}{\partial z \partial x} ; \\
& \frac{\partial^{2} \dot{\varepsilon}_{11}}{\partial y \partial z}=\frac{\partial}{\partial x}\left(-\frac{\partial \dot{\varepsilon}_{23}}{\partial x}+\frac{\partial \dot{\varepsilon}_{12}}{\partial z}+\frac{\partial \dot{\varepsilon}_{13}}{\partial y}\right) ; \\
& \frac{\partial^{2} \dot{\varepsilon}_{22}}{\partial z \partial x}=\frac{\partial}{\partial y}\left(-\frac{\partial \dot{\varepsilon}_{31}}{\partial y}-\frac{\partial \dot{\varepsilon}_{23}}{\partial x}-\frac{\partial \dot{\varepsilon}_{21}}{\partial z}\right) ; \\
& \frac{\partial^{2} \dot{\varepsilon}_{33}}{\partial x \partial y}=\frac{\partial}{\partial z}\left(-\frac{\partial \dot{\varepsilon}_{12}}{\partial z}-\frac{\partial \dot{\varepsilon}_{31}}{\partial y}-\frac{\partial \dot{\varepsilon}_{32}}{\partial x}\right)
\end{aligned}
$$

Parmi lesquelles seulement une ne se résout pas de la même façon :

$$
\frac{\partial^{2} \dot{\varepsilon}_{11}}{\partial y^{2}}+\frac{\partial^{2} \dot{\varepsilon}_{22}}{\partial x^{2}}=2 \frac{\partial^{2} \dot{\varepsilon}_{12}}{\partial x \partial y}
$$

nous obtenons que les composantes des vitesses de déplacement, indépendamment du lien entre les éléments tenseurs de tensions et vitesses de déformation, sont représentées par des formules qui ont la forme de :

$$
u_{1}=q_{1}(x, y), \quad u_{2}=q_{2}(x, y), \quad u_{3}=(C+A x+B y) \cdot z
$$

Ainsi, nous avons obtenu les conditions générales concernant le champ des vitesses de déplacement $u_{i}$, assurant la réalisation de $(1),(2)$ et nous sommes arrivés à un problème de plan « généralisé ».

En utilisant une approximation par éléments-finis, nous considérerons qu'à l'intérieur de chaque élémentfini, on a les relations suivantes pour les vitesses de déplacement :

$$
u_{1}=q_{1}(x, y), \quad u_{2}=q_{2}(x, y), \quad u_{3}=C \cdot z
$$

Soient $x$ et $y$ les coordonnées des points dans le plan $z=$ const., avec $u_{1}=u$ et $u_{2}=v$, correspondant à la vitesse de déplacement.

Comme on le sait, (compte tenu de (2), (17)) les vitesses de déformations s'expriment au travers des vitesses de déplacement de la manière suivante :

$$
\begin{aligned}
& \dot{\varepsilon}_{11}=\frac{\partial u}{\partial x}, \quad \dot{\varepsilon}_{22}=\frac{\partial v}{\partial y}, \quad \dot{\varepsilon}_{33}=c, \quad \dot{\varepsilon}_{12}=\frac{1}{2} \dot{\gamma}_{12}, \\
& \dot{\gamma}_{12}=\frac{\partial u}{\partial y}+\frac{\partial v}{\partial x}, \quad \dot{\varepsilon}_{13}=\dot{\varepsilon}_{23}=0
\end{aligned}
$$

La relation entre les contraintes et les vitesses de déformation, caractéristique de l'état physique du milieu 
dans des conditions de superplasticité [1], s'exprime de la façon suivante :

$$
\sigma_{i j}=\mu\left(u_{i, j}+u_{j, i}\right)+\left[\left(K \cdot \Delta t-\frac{2}{3} \mu\right) u_{\mathrm{p}, \mathrm{p}}+\sigma^{*}\right] \delta_{i j}
$$

Nous constatons que :

$$
\sigma_{31}=\sigma_{32}=0, \quad \sigma_{33}=2 \mu \cdot c+\left(K \cdot \Delta t-\frac{2}{3} \mu\right) \cdot \dot{\theta}+\sigma^{*}
$$

Ici le terme $\dot{\theta}$ caractérise la vitesse de changement de volume :

$$
\dot{\theta}=\dot{\varepsilon}_{11}+\dot{\varepsilon}_{22}+\dot{\varepsilon}_{33}=3 \dot{\varepsilon}=\frac{\partial u}{\partial x}+\frac{\partial v}{\partial y}+c
$$

avec $K$ - facteur de compression triaxiale ; $\mu$ - facteur apparent de viscosité, qui est une fonction de l'intensité de la vitesse de déformation et dépend d'un certain nombre de paramètres physiques $\mu=\mu\left(\dot{\varepsilon}_{\mathrm{e}}\right) ; \sigma^{*}$ - pression hydrostatique accumulée.

En prenant en compte les conditions limites de tensions, les vitesses de déplacement et les conditions de contact, les équations de la méthode des éléments-finis permettant de résoudre le changement de forme quasistatique des couvercles dans des conditions de superplasticité peuvent être écrites en suivant la méthode exposée en détails dans les travaux référencés [1-3].

\section{Séquencement des opérations à réaliser pour le calcul de la déformation dans la section critique de l'enveloppe}

Tout d'abord, il faut, d'après la configuration de l'outillage, déterminer le sens de l'étirage maximum moyen et celui de l'étirage minimum moyen. Pour le système de coordonnées, il est recommandé de choisir pour $\mathrm{O} z$ le sens de l'étirage minimum relatif, avec

$$
\ln \frac{l}{l_{0}} \rightarrow \min
$$

où $l_{0}$ - est la dimension initiale caractéristique de la section; $l$ - la longueur de la ligne moyenne du couvercle qui se forme à la section considérée après formage (ou la longueur du pourtour correspondant à la section de la matrice).

La direction $\mathrm{O} x$ est choisie comme la plus caractéristique ou la plus dangereuse du point de vue de la probabilité de perte de continuité au cours du formage ou de l'étirage maximum. L'axe $\mathrm{O} y$ est choisi pour le sens de l'action de la pression $P(t)$ et de la déformation du couvercle (Fig. 1).

Ensuite, à l'intersection $(x, y)$, il faut définir la valeur de $x^{*}$, dont le voisinage $\left(x^{*}-\omega, x^{*}+\omega\right)$ sera contrôlé directement par le calcul. On définit également les limites de fixation du couvercle à la section $(x, y):\left(x_{\min }, 0\right)$ et $\left(x_{\max }, 0\right)$.

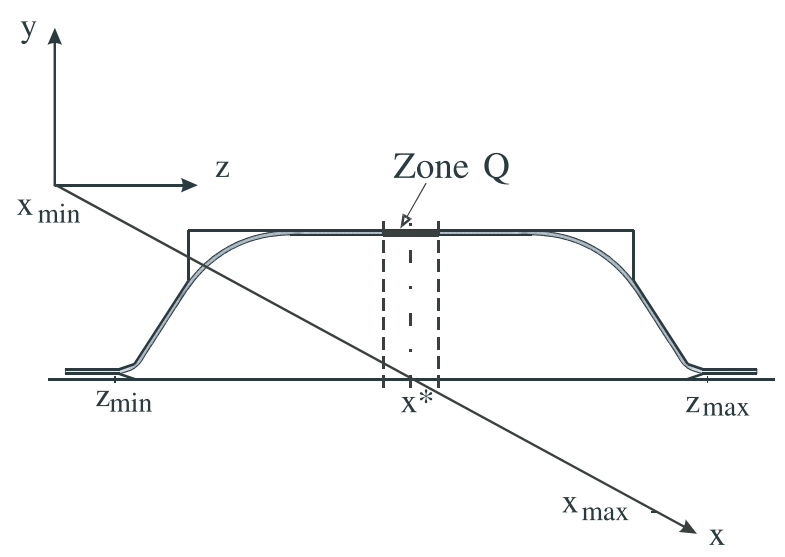

Fig. 2. Définition de la zone $Q$ dans le voisinage du point $x^{*}$ pour le calcul des déformations moyennes $\varepsilon_{33}^{*}(h(t))$ pour $\forall x \in O_{\Delta}\left(x^{*}\right)$.

Puis on choisit $k$ des points $x_{i} \in\left[x_{\min }, x_{\max }\right], i=$ $1,2, \ldots, k$. On fait passer par ces points des plans perpendiculaires à l'axe $\mathrm{O} x$. Puis on calcule les déformations moyennes obtenues à ces sections

$$
\varepsilon_{33}\left(x_{i}\right)=\ln \frac{l_{i}}{l_{i}^{0}}
$$

où $l_{i}^{0}$ - est la longueur initiale de la section $i$ du couvercle; $l_{i}$ - la longueur du pourtour correspondant à la section de la matrice.

Grâce à la valeur de $x^{*}$ on trouve $x_{j}$, pour laquelle $\varepsilon_{33}\left(x_{j}\right)=\varepsilon_{33}\left(x^{*}\right)$, et on normalise de la façon suivante :

$$
q\left(x_{i}\right)=\frac{\varepsilon_{33}\left(x_{i}\right)}{\varepsilon_{33}\left(x^{*}\right)}, \quad q\left(x^{*}\right)=1
$$

D'ailleurs, en général, les coordonnées $x_{j}$ et $x^{*}$ peuvent être confondues.

Ensuite, il faut résoudre le plan à l'intersection de la matrice et du couvercle par le plan $(y, z)$ au point $x=x^{*}$.

Le calcul de la déformation s'effectue à l'aide de la méthode des éléments-finis par le progiciel SPLEN [4-6]. Dans la zone $Q$ définie (Fig. 2) on calcule les déformations moyennes $\varepsilon_{33}$ en fonction de la hauteur $h$. Pendant le traitement, la hauteur $h$ varie entre 0 et $h_{\max }$ (la hauteur $h_{\text {max }}$ est limitée par la profondeur de la matrice).

Ainsi, on obtient la fonction :

$$
\varepsilon_{33}^{*}(h(t))=\left.\frac{\sum_{e \in e^{*}} \varepsilon_{33}^{\mathrm{e}} \cdot s^{\mathrm{e}}}{\sum_{e \in e^{*}} s^{\mathrm{e}}}\right|_{h=h(t)}, \quad e^{*} \in O_{\Delta}\left(x^{*}\right)
$$

dans laquelle $s^{\mathrm{e}}$ sont les superficies correspondantes des triangles de l'approximation par éléments-finis au voisinage de $O_{\Delta}\left(x^{*}\right)$.

La courbe établie $\varepsilon_{33}^{*}(h(t))$ permet de préciser les valeurs $\varepsilon_{33}\left(x_{i}\right)$, obtenues précédemment par la géométrie, ainsi que de lier ces valeurs à la hauteur courante du dôme.

En effet, étant donné que

$$
\varepsilon_{33}^{*}(h(t)) \equiv \varepsilon_{33}^{*}\left(x^{*}, h(t)\right),
$$


alors

$$
\varepsilon_{33}\left(x_{i}, h(t)\right)=\varepsilon_{33}^{*}\left(x^{*}, h(t)\right) \cdot q(x) .
$$

Pour un calcul par ordinateur, il est intéressant de discrétiser ce dernier rapport en fonction des valeurs des coordonnées $k: x_{1}, x_{2}, \ldots, x_{k}$ et des valeurs $n$ des hauteurs : $h_{1}, h_{2}, \ldots, h_{n}$.

Dans le même temps, on pose que $\varepsilon_{33} \equiv 0$ pour tout $x<x_{1}$ et $x>x_{k}$. De toute évidence, dans ces zones nous avons également, $\dot{\varepsilon}_{33} \equiv 0$.

Admettons que $\varepsilon_{33}$, suite à la numérisation effectuée, ne change pas sur le segment $\left[\frac{x_{i-1}+x_{i}}{2}, \frac{x_{i}+x_{i+1}}{2}\right]$ et est égal à $\left.\varepsilon_{33}\left(x_{i}\right)\right|_{h}$. Tandis que sur le segment $\left[h_{i}, h_{i+1}\right]$ la fonction $\varepsilon_{33}$ évolue de façon linéaire. alors

Si $x_{m} \in\left[\frac{x_{i-1}+x_{i}}{2}, \frac{x_{i}+x_{i+1}}{2}\right]$, tandis que $h \in\left[h_{i}, h_{i+1}\right]$,

$$
\begin{aligned}
\varepsilon_{33}\left(x_{m}, h\right) & =\frac{\varepsilon_{33}\left(x_{m}, h_{i+1}\right)-\varepsilon_{33}\left(x_{m}, h_{i}\right)}{h_{i+1}-h_{i}} \cdot h \\
& +\frac{\varepsilon_{33}\left(x_{m}, h_{i}\right) \cdot h_{i+1}-\varepsilon_{33}\left(x_{m}, h_{i+1}\right) \cdot h_{i}}{h_{i+1}-h_{i}}
\end{aligned}
$$

Ainsi, on peut également définir à tout moment le champ des vitesses des déformations $\dot{\varepsilon}_{33}(x, t)$.

La solution obtenue sera continue à tous les points intérieurs de l'approximation par éléments-finis. Les discontinuités, dans la solution par la troisième composante, le long de l'axe $\mathrm{O} z$, ne peuvent se trouver qu'en limite des éléments. En moyenne sur l'ensemble, les erreurs commises ne sont pas si importantes.

\section{Exécution des calculs de la pièce-test creuse et comparaison des prévisions obtenues et des résultats de l'expérience}

La vue d'ensemble de l'outillage, ainsi que le plan correspondant de la matrice assemblée sont présentés sur la figure 3 .

Suite aux considérations précédemment présentées, nous choisissons comme sens de l'axe $\mathrm{O} Z$ celui qui se trouve le long de l'échancrure longitudinale de la matrice, et l'axe $O X$ perpendiculaire à l'axe $O Z$ qui passe par l'intersection centrale de la matrice. Pour les points correspondants $x_{i}$ en fonction de la forme géométrique de l'outillage, nous définissons les longueurs initiales de la section $i$ du couvercle $l_{i}^{0}$, la longueur du pourtour correspondant de la section de la matrice $l_{i}$, la déformation $\varepsilon_{33}\left(x_{i}\right)=\ln \frac{l_{i}}{l_{i}^{0}}$, et les valeurs normalisées $q\left(x_{i}\right)$ d'après le rapport (23).

Résumons toutes les mesures et tous les calculs dans le tableau 1.

Ensuite, nous choisissons une autre section de contrôle le long de l'axe $\mathrm{O} z$, avec $x=x^{*}=63 \mathrm{~mm}$. En plan, cette section a la forme présentée sur la figure 4.

Dans cette section, on calcule la déformation de la section plane du couvercle en conditions de maintien de l'état d'écoulement superplastique moyen en volume $[7,8]$.
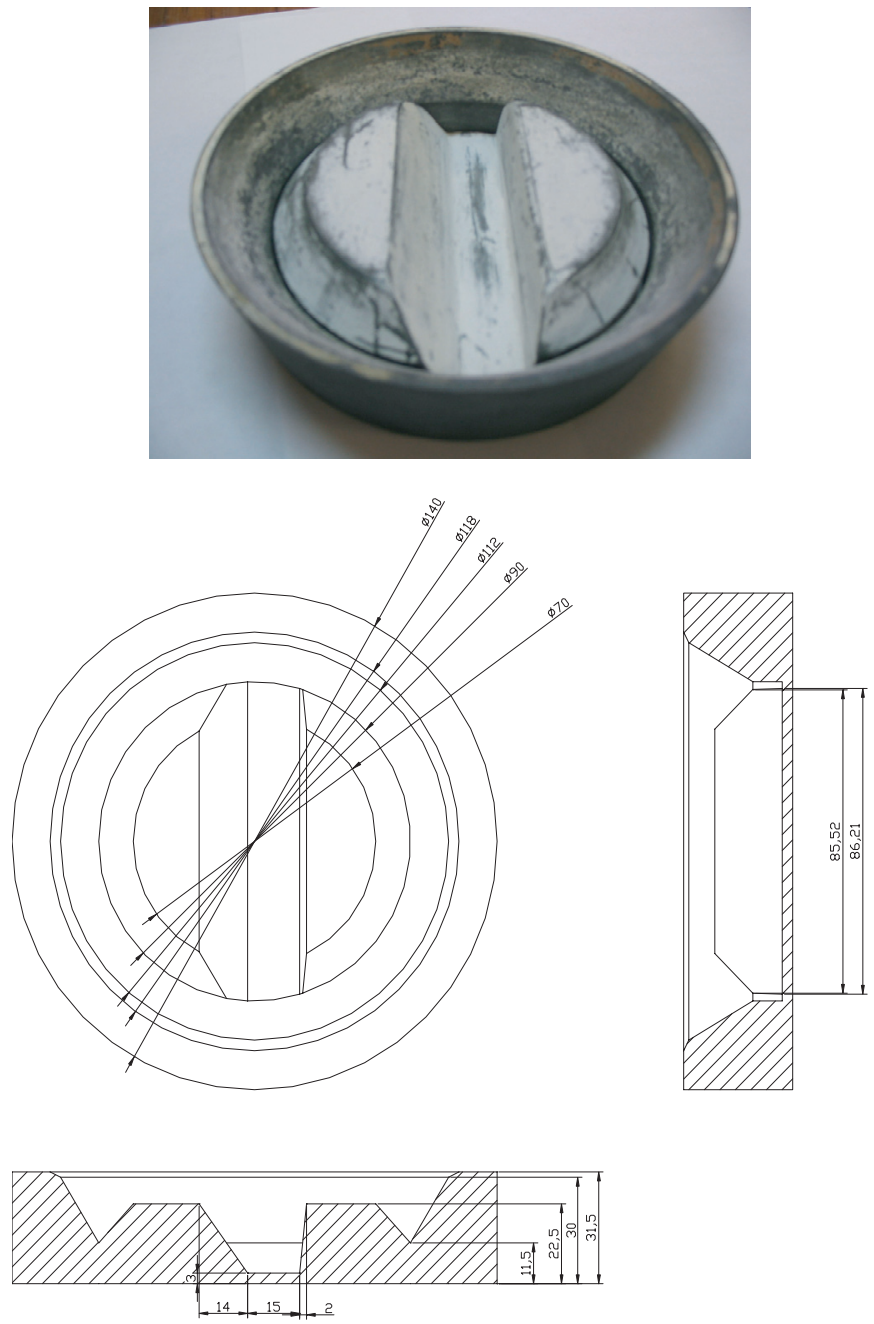

Fig. 3. Vue d'ensemble et plan de la matrice destinée à la pièce test.

Le régime contrôlé de formage s'effectue sans limite de la valeur maximale de pression $\left(P_{\max }\right)$ pour une température de $T=825{ }^{\circ} \mathrm{C}$. Dans le processus de calcul de formage (Fig. 5), on enregistre la fonction $\varepsilon_{33}\left(x^{*}, h\left(z_{0}, t\right)\right)$, définie au voisinage de $z=z_{0}$.

Maintenant, après avoir utilisé l'équation (24), remplissons une matrice élément spéciale $\varepsilon_{33}\left(x_{i}, h_{j}\right), i=$ $1,2, \ldots, k ; j=1,2, \ldots, n$ (Tab. 2 ).

Dès que le champ de déformation $\varepsilon_{33}\left(x_{i}, h_{j}\right)$ est obtenu, on peut passer aux calculs de prévision de la déformation dans la section analysée $z=z_{0}$.

On calcule le régime optimal de pression $P(t)$ assurant au couvercle un état de superplasticité au voisinage de $x=x^{*}$ ou sur l'ensemble de la section [9]. La pression trouvée de cette manière peut être recommandée pour assurer les conditions de superplasticité. La correspondance entre la prévision faite et le processus réel de formage sera d'autant plus fine que les axes des coordonnées auront été correctement choisis et la matrice élément $\varepsilon_{33}$ bien déterminée.

Dans le calcul de la pression, il a été pris en compte une limitation en valeur maximale $P_{\max }=1,5 \mathrm{MPa}$, de 
Tableau 1. Caractéristiques géométriques de la matrice.

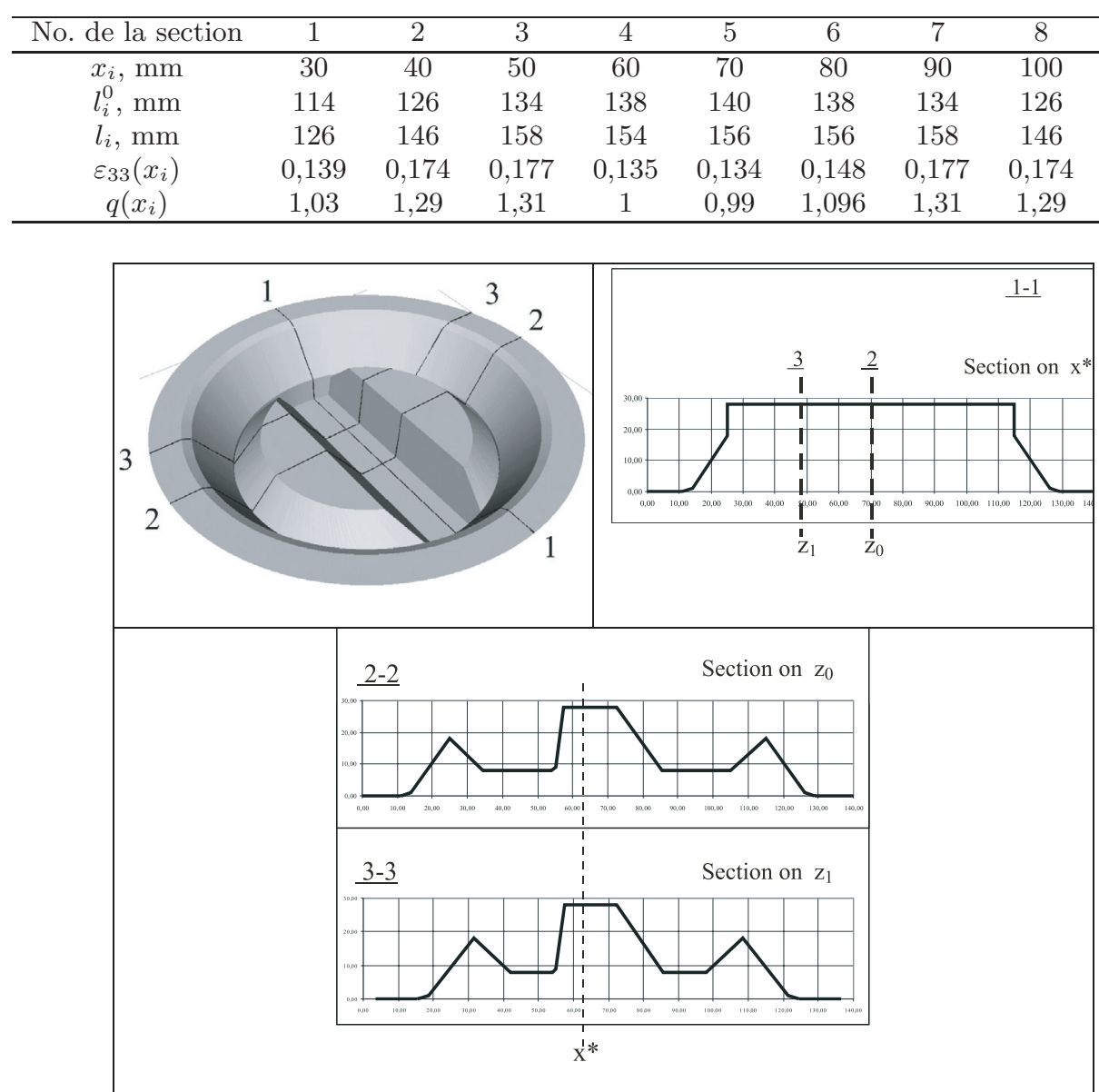

Fig. 4. Choix des sections contrôlées dans la pièce. 1-1 - section auxiliaire le long de l'axe $\mathrm{O} Z$ pour $x=x^{*}=63 \mathrm{~mm} ; 2-2-$ section contrôlée (axiale) le long de l'axe $\mathrm{O} x$ pour $z=z_{0}=70 \mathrm{~mm} ; 3-3$ - section contrôlée (complémentaire) le long de l'axe O $x$ pour $z=z_{1}=47,5 \mathrm{~mm}$.
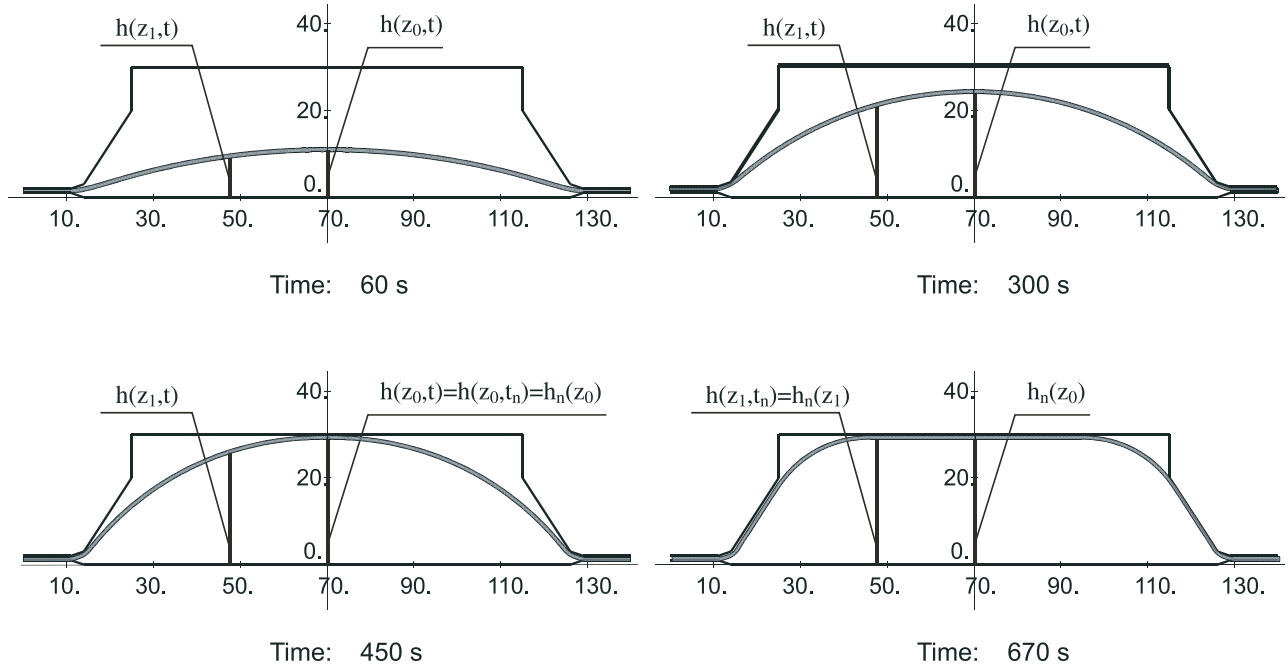

Fig. 5. Déformation de la section longitudinale de l'enveloppe avant qu'elle ne touche les points de contrôle $z=z_{0}$ et $z=z_{1}$. 
Tableau 2. Champ de déformation $\varepsilon_{33}\left(x_{i}, h_{j}\right)$ pour le couvercle de la pièce creuse pour $z=z_{0}$.

\begin{tabular}{|c|c|c|c|c|c|c|c|}
\hline$x_{x_{i}, \mathrm{~mm}} h_{j}, \mathrm{~mm}$ & 0,0 & 5,0 & 10,0 & 15,0 & 20,0 & 25,0 & 29,0 \\
\hline 20 & 0,0 & 0,003089 & 0,015444 & 0,036758 & 0,065999 & 0,102242 & 0,135911 \\
\hline 35 & 0,0 & 0,003867 & 0,019333 & 0,046013 & 0,082618 & 0,127987 & 0,170133 \\
\hline 45 & 0,0 & 0,003933 & 0,019667 & 0,046807 & 0,084042 & 0,130193 & 0,173067 \\
\hline 55 & 0,0 & 0,003 & 0,015 & 0,0357 & 0,0641 & 0,0993 & 0,132 \\
\hline 65 & 0,0 & 0,002978 & 0,014889 & 0,035436 & 0,063625 & 0,098564 & 0,131022 \\
\hline 75 & 0,0 & 0,003289 & 0,016444 & 0,039138 & 0,070273 & 0,108862 & 0,144711 \\
\hline 85 & 0,0 & 0,003933 & 0,019667 & 0,046807 & 0,084042 & 0,130193 & 0,173067 \\
\hline 95 & 0,0 & 0,003867 & 0,019333 & 0,046013 & 0,082618 & 0,127987 & 0,170133 \\
\hline 105 & 0,0 & 0,003089 & 0,015444 & 0,036758 & 0,065999 & 0,102242 & 0,135911 \\
\hline 120, & 0,0 & 0,0 & 0,0 & 0,0 & 0,0 & 0,0 & 0,0 \\
\hline
\end{tabular}
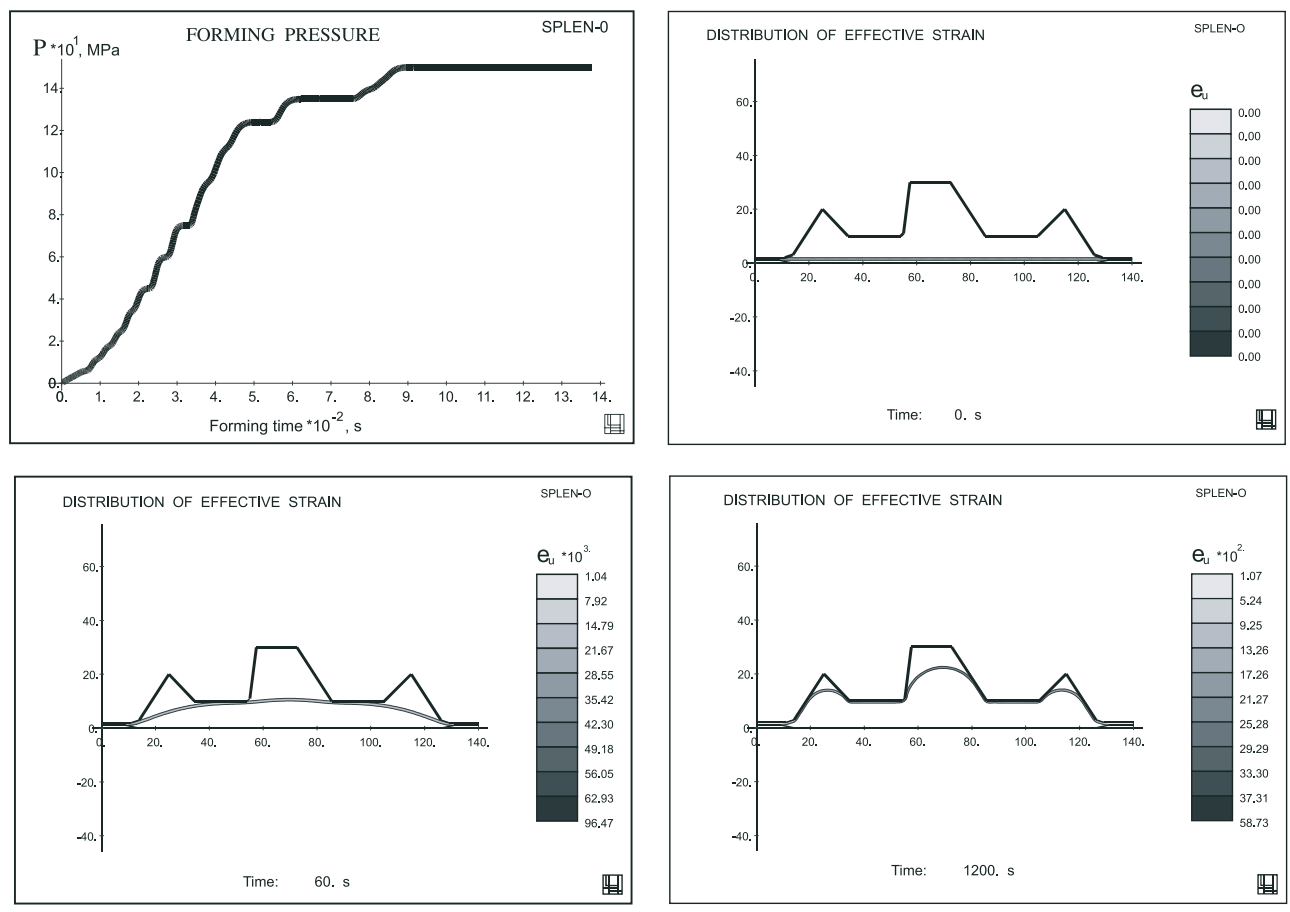

Fig. 6. Courbe de pression et déformation de la section du couvercle pour $z=z_{0}$.

façon à permettre au système de mesure de l'entraînement de fonctionner de façon fiable.

La courbe de pression obtenue et le formage par étape de la section pour $z=z_{0}$ sont présentés en figure 6 .

Le contrôle expérimental (Fig. 7) a montré que les écarts de forme extérieure de la section du couvercle obtenue de façon expérimentale, par rapport à la prévision faite, se situent dans une limite de $0,5 \mathrm{~mm}$, ce qui est un excellent résultat, puisque cela ne dépasse pas $50 \%$ de l'épaisseur initiale du couvercle (Fig. 8b).

Le couvercle formé a été coupé. Les mesures correspondantes de son épaisseur ont été effectuées à des points caractéristiques. Les résultats des mesures et de la prévision théorique sont présentés sur la figure 8.

Le taux d'erreur de la prévision de l'épaisseur se trouve dans les limites de $5 \%$ de l'épaisseur initiale du couvercle moulé.

De notre point de vue, la solution obtenue à la section $z=z_{1}=47,5 \mathrm{~mm}$ présente un intérêt tout particulier.

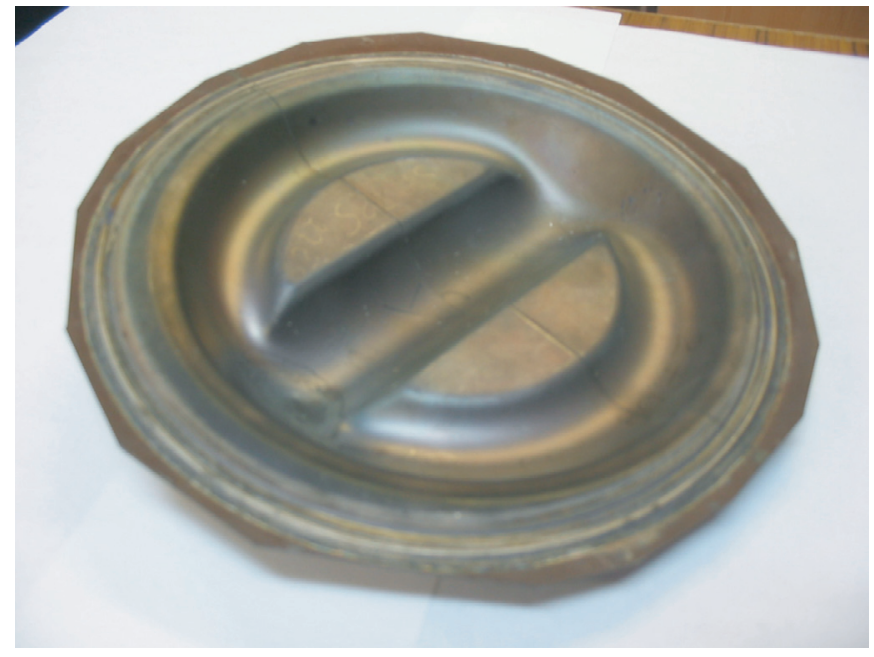

Fig. 7. Couvercle obtenu par formage expérimental. 


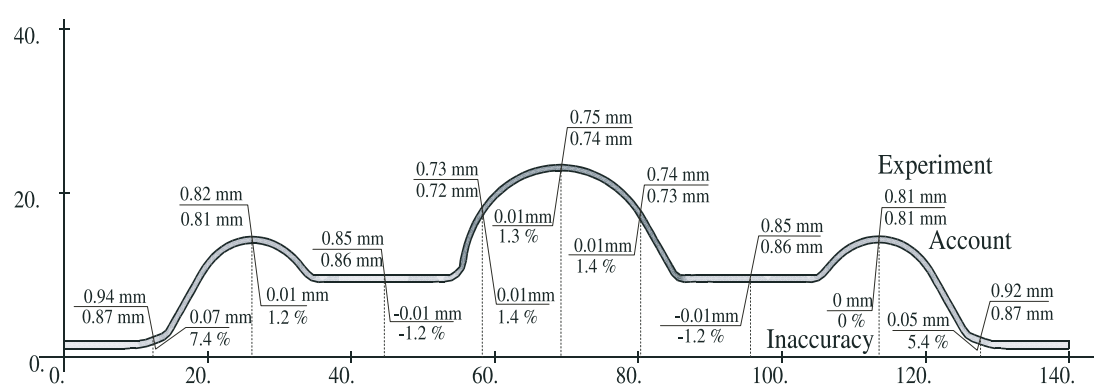

a)

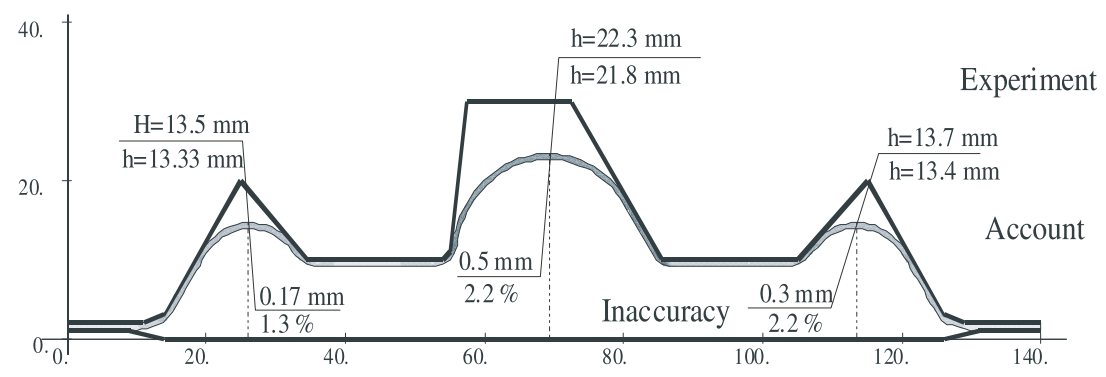

b)

Fig. 8. Épaisseur du couvercle après formage et prévision correspondante (a), définition théorique et expérimentale de la forme du couvercle (b) $\left(T=825^{\circ} \mathrm{C}, t=1200 \mathrm{~s}, P=P(t), z=z_{0}\right)$.

Pour ce cas, le champ de déformation $\varepsilon_{33}$ sera différent, puisque la fonction $\varepsilon_{33}\left(x^{*}, h\left(z_{1}, t\right)\right)$ se trouvera dans le voisinage de $z=z_{1}$, tandis que $q(x)$ restera inchangée.

La prévision de déformation effectuée, puis sa comparaison avec les données obtenues de façon expérimentale, confirment une bonne convergence. Les écarts, sur la configuration externe et sur les différences d'épaisseur n'excèdent pas ceux obtenus précédemment.

\section{Conclusion}

L'algorithme proposé pour une analyse par approximation du formage superplastique des couvercles, à l'aide du calcul des sections critiques en plans, peut être utilisé pour l'évaluation rapide et l'optimisation des processus technologiques étudiés afin d'obtenir des structures de forme couvercle, dans des conditions de superplasticité.

Ce travail a été réalisé à la demande et avec le soutien de la compagnie internationale European Aeronautic Defence and Space Company, Airbus SA et de la société KOMMEK Ltd. (Russie).

\section{Références}

[1] E.N. Chumachenko, Simulation mathématique de l'emboutissage des couvercles dans des conditions de superplasticité, M : MGIEM, 1999, 158 p.

[2] E.N. Chumachenko, Simulation mathématique de l'emboutissage plastique des matériaux par traitement sous pression, M : MGIEM, 1998, 157 p.
[3] V.S. Davydov, E.N. Chumachenko, Méthode de réalisation d'une interférence de contact en MEF pour résoudre le problème de l'emboutissage de matériaux pleins, Izvestia de l'Académie des Sciences russe (Nouvelles de l'Académie des Sciences de Russie, Mécanique des Solides), MTT 4 (2000) 53-56

[4] E.N. Chumachenko, M.A. Tsepin, L.E. Rogalevich, Calcul des procédés d'emboutissage et calcul des éléments de l'outillage, du matériel et de l'équipement à l'aide du progiciel « SPLEN », J. construction mécanique 4 (2000) 37-45

[5] E.N. Chumachenko, O.M. Smirnov, Computer aided design of superplastic forming processes based on the SPLEN program set, ICSAM'94, Materials Science Forum 170-172 (1994) 601-606

[6] E.N. Chumachenko, O.M. Smirnov, S.E. Chumachenko, Stress-strain state of a blank in superplastic bulk forming under mutual influence of material rheology and contact friction, ICSAM'97, Materials Science Forum 243245 (1997) 745-750

[7] E.N. Chumachenko, S.E. Chumachenko, Simulation mathématique des régimes de pression permettant l'emboutissage de couvercles à viscosité non-linéaire dans des conditions locales d'écoulement superplastique, Izvestia de l'Académie des Sciences russe, MTT 6 (2000) 134-142

[8] E.N. Chumachenko, V.K. Portnoï, S.A. Axionov, D.S. Rylov, Emboutissage superplastique des couvercles en titane dans une gamme étendue de températures, Nauka proizpodstvu, La science pour la production 12 (2003) $2-6$

[9] E.N. Chumachenko, S.E. Chumachenko, Brevet No. 2226442, Méthode d'emboutissage au gaz des couvercles dans des conditions d'isothermie et de superplasticité de l'écoulement du métal (priorité du 29 mai 2003) 